\title{
ESTIMATIVA DA BIOMASSA FOLIAR E POTENCIAL EXTRATIVISTA DA PIMENTA Pseudocaryophyllus (CATAIA)
}

\author{
ESTIMATE OF FOLIAR BIOMASS AND EXTRATIVIST POTENTIAL OF PIMENTA \\ PSEUDOCARYOPHYLLUS (CATAIA)
Rozane Loyola Eisfeld', Roni Djeison Ansolin², Amanda Silva Rocha D'Angelis ${ }^{3}$, Edson Rangel Silva Júnior', Sérgio Vinícius Serejo da Costa Filho ${ }^{5}$ \\ 1, 2, 3, 5 Universidade Federal do Paraná, Programa de Pós-graduação em Engenharia Florestal, Curitiba, \\ Paraná, Brasil - rozaneloyolaeisfeld@hotmail.com; roni_ansolin@hotmail.com; \\ amandadangelis@hotmail.com \&sergio.vscf@gmail.com \\ ${ }^{4}$ Instituto Chico Mendes de Conservação da Biodiversidade (ICMBio), Campo Largo, Paraná, Brasil - \\ edson.junior@icmbio.gov.br \\ RESUMO \\ Pimenta pseudocaryophyllus (Gomes) Landrum (cataia) é uma espécie da família Myrtaceae de importância \\ econômica e cultural. Suas folhas são consideradas um produto florestal não madeireiro (PFNM), sendo extraídas \\ para produção de aguardente, conhecida como "whisky caiçara". O presente estudo teve como objetivo testar \\ modelos matemáticos com a finalidade de gerar estimativas da biomassa seca foliar da espécie. Foram utilizados \\ dados de biomassa seca foliar de 23 indivíduos de cataia, obtidos por meio de determinações diretas pelo método \\ destrutivo. Todas as árvores amostradas foram medidas quanto ao CAP (circunferência à altura do peito), diâmetro e \\ altura de copa, altura total e número de brotações. Os resultados mostraram que o melhor modelo, empregando a \\ variável número de brotações, obteve um erro de estimativa de $16 \%$, já os demais, obtiveram erros superiores a $30 \%$. \\ Por fim, o modelo Stepwise Completo apresentou o melhor desempenho para a variável dependente biomassa seca \\ das folhas. Ainda, a extração das folhas de P. pseudocaryophyllus é uma atividade econômica e cultural das famílias \\ tradicionais da comunidade de Ilha Comprida, cujo manejo sustentável pode contribuir na perpetuação e preservação \\ da espécie.
}

PALAVRAS-CHAVE: Área de Preservação Ambiental, Equações Volumétricas, Ilha Comprida, Plano de manejo, Produto Florestal Não Madeireiro.

\section{ABSTRACT}

Pimenta pseudocaryophyllus (Gomes) Landrum (cataia) is a species of Myrtaceae family, with economic and culturally importance. Its leaves are considered a non-timber forest product (NTFP), being extracted for production of brandy, known as "caiçara whiskey". The present study aimed to test mathematical models with the purpose of generating estimates of the dry leaf biomass of the species. Data of dry leaf biomass of 23 cataia individuals were obtained through direct determinations by the destructive method. All trees sampled were measured for $\mathrm{CBH}$ (circumference at breast height), diameter and height of the tree top, total height and number of buds. The results showed that the best model, using the number of buds, obtained an error of $16 \%$, while the other models had errors above $30 \%$. All in all, the model Stepwise Completo showed the best performance for the dependent variable of dry leaf biomass. Also, the extraction of $P$. pseudocaryophyllus leaves is an important economic and cultural activity for the traditional families of the Ilha Comprida community, whose sustainable management can to the contribute preservation of the specie.

KEYWORDS: Environmental Preservation Areas, Volumetric Equations, Management plan, Non-Timber Forest Product. 


\section{INTRODUÇÃO}

Existe no mundo um uso intensivo dos recursos florestais madeireiros. Sabe-se, porém, dos danos aos ecossistemas naturais causados por esta forma de utilização da floresta. Entretanto, a floresta pode conceder outros produtos que não madeira, muitos dos quais são utilizados por populações locais e até mesmo explorados comercialmente há séculos (SANTOS et al., 2003).

Estes produtos são denominados produtos florestais não madeireiros (PFNM), correspondendo a qualquer material biológico, que não madeira ou derivado, extraído de florestas naturais ou manejada, com fins domésticos ou comerciais, com ou sem significância social, religiosa ou cultural definida (ANACLETO et al., 2017).

As folhas de Pimenta pseudocaryophyllus (Gomes) Landrum (Myrtaceae) caracterizam-se como um PFNM muito apreciado nas regiões litorâneas de São Paulo e Paraná, usado por suas propriedades medicinais e aromáticas, no preparo de chás e cachaça saborizada (PAULA et al., 2008; YOKOMIZO \& NAKAOKA-SAKITA, 2014). O extrativismo de folhas da espécie para comercialização é praticado pelas comunidades do município de Ilha Comprida (SP), assim como foi registrado para o estado do Paraná (D’ANGELIS \& NEGRELLE, 2014).

Ilha Comprida localiza-se no litoral sul de São Paulo, entre os municípios de Iguape e Cananéia. O município foi nomeado Reserva da Biosfera pela UNESCO e tem todo o seu território protegido por Unidades de Conservação de Uso Sustentável (BECEGATO \& SUGUIO, 2007). A inserção do município em UCs, interfere nas atividades econômicas das populações locais, uma vez que restringe o acesso aos recursos naturais, conforme determina o Sistema Nacional de Unidades de Conservação - SNUC (BRASIL, 2000). Entretanto, a utilização de recursos não madeireiros, que não coloquem em risco a sobrevivência das espécies, é permitida (BRASIL, 2006) e deve ser incentivada, no sentido de proporcionar alternativas de trabalho e renda a essas populações.

Tendo em vista a importância econômica da espécie $P$. pseudocaryophyllus para as comunidades de Ilha Comprida e os riscos associados ao aumento de sua escala de produção, verificou-se a necessidade de implantação de um plano de manejo, visando o uso racional da matéria prima em questão. Para tanto, além da realização de censo florestal, é importante estimar a biomassa foliar dos indivíduos amostrados, que permita determinar o potencial de coleta de folhas e gerar recomendações mais precisas ao manejo da espécie.

A respeito da determinação de biomassa, Corte et al.
(2012) afirmam que os métodos indiretos não podem ser utilizados sem o ajuste e a calibragem prévia de equações, e devem ser empregados conjuntamente com métodos diretos. Um procedimento utilizado comumente para a quantificação de biomassa é o recurso de regressão, pelo qual procede-se a derrubada dos indivíduos e a determinação da biomassa de seus componentes. Posteriormente são ajustadas equações de regressão, relacionando a biomassa às variáveis biométricas das árvores (VIANA et al., 2013).

No sentido de contribuir com a sustentabilidade do extrativismo de $P$. pseudocaryophyllus praticado em Ilha Comprida, o presente estudo tem como objetivo testar e propor modelos matemáticos de estimativa da biomassa foliar, considerando variáveis independentes, bem como quantificar a biomassa foliar de três unidades de trabalhos, visando subsidiar informações para o manejo sustentável da espécie.

\section{MATERIAL E MÉTODOS}

\section{Área de estudo}

O município de Ilha Comprida está localizado no litoral sul do Estado de São Paulo, entre os municípios de Iguape e Cananéia sendo, em 1987, declarado como Área de Proteção Ambiental. Por definição, são áreas "dotadas de atributos abióticos, bióticos, estéticos e culturais, formado por um mosaico de ecossistemas biodiversos, todos associados à Mata Atlântica (SANTOS, 2016).

A temperatura média anual do município é de $23,8^{\circ} \mathrm{C}$, variando de $19,8^{\circ} \mathrm{C}$, em julho, a $27,8^{\circ} \mathrm{C}$, em fevereiro (SILVA, 1989). Ilha Comprida é constituída por sedimentos arenosos quaternários, predominantemente holocênicos, exceto para poucos remanescentes de terraços marinhos pleistocênicos e um morrote constituído de rochas alcalinas, ambos localizados no extremo sul da Ilha (IIZUKA et al., 2011).

Apesar da ocorrência extensa de cataia dentro do município de Ilha Comprida, o estudo contemplou a Comunidade de Pedrinhas, localizada no sul da ilha.

\section{Determinação da biomassa foliar}

Os dados utilizados para a estimação dos parâmetros das equações de biomassa foram obtidos na Área do Plano de Manejo (43,6 ha), a qual está subdividida em 24 unidades de trabalho (UTs). Para a determinação da biomassa foliar foram escolhidos indivíduos presentes nas diferentes classes diamétricas, baseando-se no censo 
florestal realizados nas três UTs (6,2 ha).

Os indivíduos selecionados para determinação da biomassa $(n=23)$ tiveram medidas as seguintes variáveis dendrométricas: circunferência à altura do peito (CAP), medida com auxílio de uma fita métrica; diâmetro de copa (dc), medido com base na projeção horizontal da copa, com auxílio de fita métrica; e altura total (ht) e altura de copa (hc), medidas com auxílio de vara graduada. Contou-se, também, o número de brotações encontrados, mediante o critério de que haja mais de 15 folhas para que seja considerado uma brotação.

Para a determinação da biomassa foliar verde individual foi utilizado o método destrutivo, coletando-se $1 / 3$ do volume foliar total da árvore. Para tanto, fez-se uso de podão e serrote curvo para poda. Optou-se por não extrair a totalidade das folhas por se tratar de uma Área de Proteção Ambiental e pelo fato de que os indivíduos pertenciam às Unidades de Trabalho que compõe o plano de manejo da espécie.

Após a extração das folhas, estas foram acondicionadas em sacos plásticos identificados, que foram pesados individualmente com sua umidade natural, utilizando-se uma balança do tipo pesola, com capacidade de carga de $35 \mathrm{~kg}$.

Em seguida, as amostras das folhas foram inseridas em estufa de renovação e circulação de ar a uma temperatura constante de $50^{\circ} \mathrm{C}$ até atingir peso constante. Posteriormente, foram obtidos os pesos secos das folhas, em gramas, com auxílio de uma balança digital, com precisão de $0,01 \mathrm{~g}$.

Por fim, foi estimado o teor de umidade com a Equação 1:

$$
T u=\frac{P v-P s}{P s} \times 100
$$

Em que: Tu = teor de umidade, em porcentagem; Ps = peso seco (\%), em gramas ( $\mathrm{g}) ; \mathrm{Pv}=$ peso verde, em gramas (g).

A biomassa seca foi estimada a partir da multiplicação da biomassa verde pelo teor de umidade de cada amostra.

\section{Equações}

Devido à dificuldade de obtenção da biomassa foliar, optou-se pelo ajuste de modelos de regressão para estimar a biomassa potencial das Unidades de Trabalho pertencentes ao plano de manejo da cataia. A avaliação indireta da biomassa procura correlacionar variáveis de fácil obtenção, como o DAP, altura, diâmetro e altura de copa.

Além das variáveis dendrométricas obtidas em campo, outras variáveis independentes também foram calculadas, sendo elas, Equações 2 e 3 :

Diâmetro médio quadrático

$$
D g=2 \times \sqrt{\frac{\sum g_{1}^{2}+g_{2}^{2}+\cdots g_{n}^{2}}{\pi} \times 100}
$$

Em que: $\mathrm{Dg}$ = diâmetro médio quadrático, em $\mathrm{cm} ; \mathrm{g}=$ área transversal cada fuste, em $\mathrm{m} ; \mathrm{n}=$ número de fustes.

Volume de copa

$$
V c=\frac{4 \times \pi \times\left(\frac{d c}{2}\right)^{2} \times \frac{h c}{2}}{3}
$$

Em que: $\mathrm{Vc}=$ volume de copa, $\mathrm{em}^{3} ; \mathrm{dc}=$ diâmetro de copa, em $\mathrm{m}$; hc = altura de copa, em $\mathrm{m}$.

A análise de correlação foi realizada entre os dados de biomassa e as variáveis independentes, buscando identificar quais das variáveis melhor explicam o comportamento da biomassa. Posteriormente, foram ajustados os modelos de regressão linear para estimativa de biomassa. Para tanto, foram testados quatro modelos conforme apresentados na tabela 1.

Tabela 1. Equações volumétricas usadas para a estimativa de biomassa da cataia

\begin{tabular}{ccc}
\hline & Equações & Autor \\
\hline 1 & $B F S=\beta_{1} d c^{2}+\beta_{2} h c^{2}+\beta_{3} d c^{2} \times h c$ & Naslund \\
$+\beta_{4} d c \times h c$ & (SILVESTRE et al., 2014) \\
2 & $B F S=\beta_{0}+\beta_{1} d c^{2} \times h c$ & Spurr \\
3 & Método de escolha de variáveis (i) & (SILVESTRE et al., 2014) \\
4 & Método de escolha de variáveis (ii) & Forward Stepwise \\
\hline
\end{tabular}

Em que: $\mathrm{BFS}=$ biomassa foliar seca, em $\mathrm{kg}$; dc = diâmetro de copa, em m; $h c=$ altura comercial, em $m ; \beta 0, \beta 1, \beta 2, \beta 3, \beta 4=$ coeficientes.

O procedimento de modelagem de regressão Forward Stepwise parte da variável independente mais correlacionada com a variável dependente (biomassa seca); no passo seguinte, inclui-se mais uma variável, que apresenta a maior correlação parcial, em função do teste F.

Devido o número de brotações ser uma variável de difícil obtenção foram gerados dois modelos, ambos pelo procedimento Forward Stepwise, o primeiro considerando a variável número de brotações e o segundo sem a inclusão desta variável. Os modelos, foram assim nomeados: 
(i) Modelo Stepwise Completo (inclui número de brotações);

(ii) Modelo Stepwise (não inclui a variável número de brotações).

O modelo máximo de regressão (Completo), inserido no procedimento Forward Stepwise, considerou as seguintes variáveis independentes apresentadas na Equação 4.

BFS $=f\left(d c, h t, h c, D g, N b r o t, d c^{2} . h c, d c . h c, h t^{2}\right)$

Em que: BFS = Biomassa foliar seca, em $\mathrm{kg}$; dc = diâmetro de copa, em $\mathrm{m}$; ht = altura total, em $\mathrm{m}$; hc = altura de copa, em $\mathrm{m}$; Dg = diâmetro médio quadrático, em cm; Nbrot = número de brotações.

Para testar a qualidade do ajuste do modelo, utilizaram-se três parâmetros de comparação: o coeficiente de determinação ajustado ( $R^{2}$ ajustado), o erro padrão da estimativa (Syx), o critério de informação de Akaike (AIC), bem como a análise gráfica dos resíduos para avaliar as pressuposições do modelo. Testou-se, ainda, a presença de confundimento de preditores nos modelos (multicolinearidade), a partir da estatística Variance Inflation Factor (VIF).

\section{RESULTADOS}

\section{Modelos de Regressão}

Com o intuito de se obter a biomassa foliar total dos indivíduos de $P$. pseudocaryophyllus nas unidades de manejo em Ilha Comprida (SP), e considerando que esta é uma variável de difícil obtenção, utilizou-se a técnica de regressão linear para ajustar um modelo matemático, a partir de 23 indivíduos amostrados. Dessa maneira, calculou-se uma matriz de correlação para verificar a relação entre a biomassa seca com as demais variáveis obtidas, conforme a Tabela 2. A Tabela 3 apresenta os resultados do teste de multicolinearidade, com os valores dos fatores de inflação da variância (FIV).

Os resultados dos ajustes dos modelos de equações que exprimem a biomassa foliar da cataia, em kg, em função das variáveis independentes, são apresentados na Tabela 4. A análise gráfica de resíduos está apresentada na Figura 1.
Tabela 2. Correlação entre biomassa foliar seca (BFS) e as demais variáveis, e suas combinações, obtidas dos indivíduos de P. pseudocaryophyllus amostrados

\begin{tabular}{cccc}
\hline VAR & CORR & VAR & CORR \\
\hline BFS & 1,000 & BFS & 1,000 \\
No brot & 0,971 & $\mathbf{d c}^{\mathbf{2}} \cdot \mathbf{h c}$ & 0,975 \\
$\mathbf{d g}$ & 0,804 & $\mathbf{d c} \cdot \mathbf{h c}$ & 0,932 \\
ht & 0,785 & $\mathbf{h t}^{\mathbf{2}}$ & 0,850 \\
dc & 0,903 & $\mathbf{d c}^{\mathbf{2}}$ & 0,942 \\
hc & 0,716 & $\mathbf{h c}^{\mathbf{2}}$ & 0,712 \\
vc & 0,975 & & \\
\hline
\end{tabular}

Em que: $\mathrm{BFS}$ = biomassa foliar seca, em kg; Nbrot = número de brotações; $\mathrm{Dg}=$ diâmetro médio quadrático, em $\mathrm{cm}$; ht = altura total, em $\mathrm{m}$; dc = diâmetro de copa, em $\mathrm{m}$; hc = altura comercial, em $\mathrm{m}$; vc = volume de copa, em $\mathrm{m}^{3}$.

Tabela 3. Fatores de inflação da variância para as variáveis independentes utilizadas nos ajustes

\begin{tabular}{|c|c|c|c|c|c|c|c|}
\hline & \multirow[b]{2}{*}{ nbrot } & \multirow[b]{2}{*}{$\mathrm{dc}^{2} \cdot h \mathrm{c}$} & \multirow[b]{2}{*}{ dc.hc } & \multicolumn{2}{|l|}{ FIV } & \multirow[b]{2}{*}{$d^{2} c^{2}$} & \multirow[b]{2}{*}{ dc.hc ${ }^{2}$} \\
\hline & & & & $h t^{2}$ & dc & & \\
\hline nbrot & - & - & - & - & - & - & - \\
\hline $\mathrm{dc}^{2} \cdot \mathrm{hc}$ & 7,9 & - & - & - & - & - & - \\
\hline dc.hc & 9,2 & 9,4 & - & - & - & - & - \\
\hline$h t^{2}$ & 3,1 & 3,0 & 3,8 & - & - & - & - \\
\hline dc & 4,8 & 8,0 & 9,8 & 3,6 & - & - & - \\
\hline$d^{2} c^{2}$ & 4,4 & 23,3 & 5,0 & 2,8 & 9,9 & - & - \\
\hline dc.hc ${ }^{2}$ & 7,6 & 4,8 & 17,7 & 2,6 & 3,6 & 2,7 & - \\
\hline
\end{tabular}

Em que: $\mathrm{VIF}=$ fator de inflação da variância; nbrot = número de brotações; $\mathrm{Dg}=$ diâmetro médio quadrático, em $\mathrm{cm} ; \mathrm{ht}=$ altura total, em $\mathrm{m} ; \mathrm{dc}=$ diâmetro de copa, em $\mathrm{m}$; hc = altura comercial, em m; vc = volume de copa, em $\mathrm{m}^{3}$.

Tabela 4. Resultados do ajuste das equações de regressão

\begin{tabular}{|c|c|c|c|c|c|}
\hline \multirow{2}{*}{\multicolumn{2}{|c|}{ Estatísticas }} & \multicolumn{4}{|c|}{ Modelos de Regressão } \\
\hline & & 1 & 2 & 3 & 4 \\
\hline \multirow{7}{*}{ 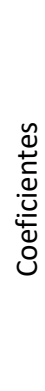 } & $\beta 0$ & - & $-0,0130$ & 0,0455 & 0,2390 \\
\hline & \multirow{2}{*}{$\beta 1$} & \multirow{2}{*}{$-0,1409$} & 0,0460 & 0,0310 & 0,0475 \\
\hline & & & $* * *$ & $* * *$ & $* * *$ \\
\hline & $\beta 2$ & $\begin{array}{c}0,0934 \\
* *\end{array}$ & - & $\begin{array}{c}0,0068 \\
* * *\end{array}$ & $\begin{array}{c}0,0155 \\
* *\end{array}$ \\
\hline & \multirow[b]{2}{*}{$\beta 3$} & \multirow[b]{2}{*}{$-0,0368$} & \multirow[b]{2}{*}{ - } & -0.1162 & -0.3496 \\
\hline & & & & $\begin{array}{c}-0,1102 \\
* * *\end{array}$ & $\begin{array}{l}-0,5450 \\
*\end{array}$ \\
\hline & $\beta 4$ & 0,0891 & - & $\begin{array}{c}0,0107 \\
* * *\end{array}$ & - \\
\hline \multirow{4}{*}{ 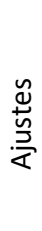 } & $\begin{array}{c}\mathrm{R}^{2} \\
\text { ajustado }\end{array}$ & 0,965 & 0,785 & 0,988 & 0,958 \\
\hline & Syx (\%) & 32,78 & 68,42 & 16,41 & 30,23 \\
\hline & AIC & $-21,62$ & 8,22 & $-51,47$ & $-38,41$ \\
\hline & $\mathrm{F}$ & 160,0 & 81,2 & 439,7 & 168,1 \\
\hline
\end{tabular}

***significativo a $1 \%, * *$ significativo a $5 \%$, significativo a $10 \%$. 


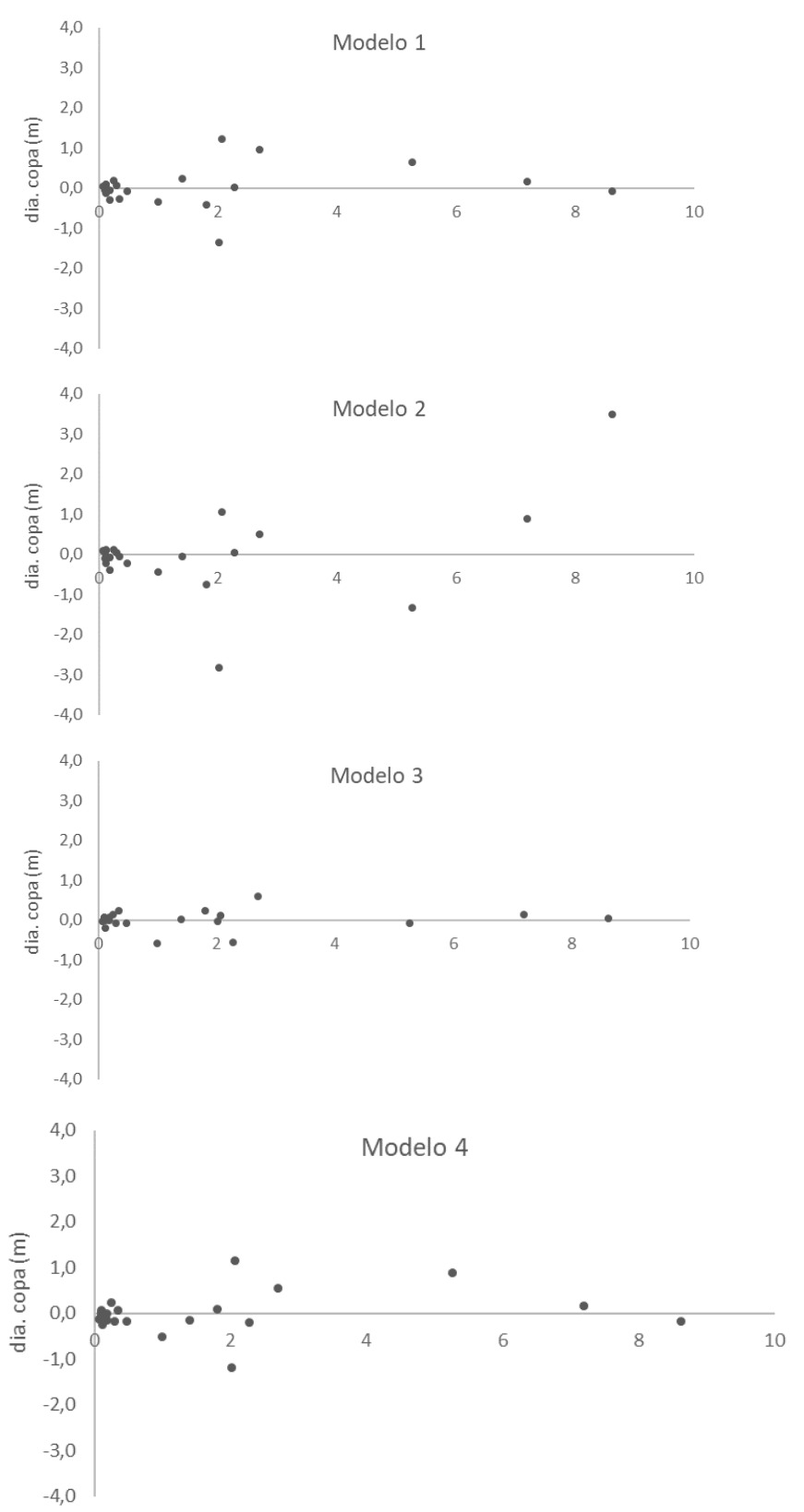

Figura 1. Gráfico de resíduos das equações de regressão.

\section{Potencial de Biomassa Foliar}

Com base nos dados obtidos no censo florestal realizado nas três Unidades de Trabalho em Ilha Comprida (SP), e no modelo matemático utilizado para estimar a biomassa seca, foi possível fazer uma estimativa da produção foliar total, que auxiliará os produtores em seu planejamento das atividades de colheita.

A biomassa foliar seca total (14,5\% umidade) existente nos 6,2 ha presentes nas três UTs totalizou aproximadamente 110 quilos, conforme apresentado na Tabela 5.
Tabela 5. Biomassa seca total estimada para cada uma das Unidades de Trabalho amostradas

\begin{tabular}{ccccc}
\hline Estimadores & UT1 & UT2 & UT3 & Total \\
\hline Biomassa Total (kg) & 19,00 & 59,50 & 31,2 & 109,70 \\
\hline Biomassa (kg/ha) & 10,40 & 35,50 & 11,6 & 17,70 \\
Média/arv. (kg) & 0,40 & 0,88 & 0,27 & 0,48 \\
№ folhas & 50.451 & 155.633 & 81.622 & 287.705 \\
№ garrafas (1 litro) & 4.749 & 14.878 & 7.798 & 27.425 \\
\hline
\end{tabular}

\section{DISCUSSÃO}

A matriz de correlação simples é um importante instrumento para verificar o grau de associação entre as variáveis dependentes e independentes com a variável dependente, neste caso a biomassa foliar. De tal forma que as variáveis independentes que apresentaram melhor correlação com a biomassa foliar foram: volume de copa $(0,975)$, número de brotações $(0,971)$ e diâmetro de copa $(0,903)$.

Os modelos 3 (Stepwise completo), 4 (Stepwise) e 1 (Naslund) apresentaram os melhores coeficientes de determinação ajustado e erro padrão da estimativa em porcentagem, sendo que modelo Stepwise completo apresentou o melhor resultado com 0,988 de coeficiente de determinação ajustado, um erro padrão da estimativa de $16,41 \%$, um AIC de $-51,47$ e a melhor distribuição de resíduos. Tal resultado pode ser explicado pelo fato deste modelo incluir o número de brotações como variável independente.

A formulação matemática resultante do ajuste pelo método "Forward Stepwise" foi:

(i) Modelo Stepwise Completo (melhores resultados, porém exige a variável número de brotações):

$$
\begin{gathered}
\text { BFS }=0,045+0,0310 \times \mathrm{dc}^{2} \times \mathrm{hc}+0,0068 \times \mathrm{Nbrot}- \\
0,1162 \times \mathrm{dc} \times \mathrm{hc}+0,0107 \times \mathrm{ht}^{2}
\end{gathered}
$$

(ii) Modelo Stepwise (adotado no estudo):

$$
\begin{gathered}
\text { BFS }=0,2390+0,0475 \times d^{2} \times h c+0,0155 \times h t^{2}-0,3496 \\
\times d c
\end{gathered}
$$

Por meio do teste de multicolinearidade indentificou que poderão existir problemas nas estimativas dos modelos de regressão, pois alguns fatores de inflação da variância foram acima de dez. Dentre os modelos, o Naslund apresentou, nas variáveis independentes, aquelas com os maiores efeitos de multicolinearidade.

Devido à dificuldade em coletar a variável número de brotações dos indivíduos de cataia optou-se em quantificar 
a biomassa foliar através do Modelo Stepwise (sem inclusão da variável "número de brotos"), uma vez que este também apresenta coeficientes robustos, garantindo uma estimativa confiável.

O ajuste final adotado, baseado no modelo Stepwise, apresentou um coeficiente de determinação ajustado de 0,958, o erro padrão da estimativa de $30,23 \%(0,484 \mathrm{~kg})$ e resíduos sem tendenciosidades.

Barbeiro et al. (2009), ajustaram equações para estimativa da biomassa de Nectandra grandiflora (canelaamarela), e obtiveram para o compartimento folhagem, um coeficiente de determinação entre $63 \%$ e $79 \%$, e um erro padrão alto, da ordem de 45 a $60 \%$, revelando um nível baixo de precisão nas estimativas de biomassa foliar com todos os modelos testados. Segundo os autores, tal imprecisão foi devida à irregularidade na arquitetura das copas da espécie.

Estima-se, também, um potencial de $500 \mathrm{~g}$ por indivíduo de cataia, considerando o peso seco foliar médio $(0,3824 \mathrm{~g})$, as três unidades somam um total de aproximadamente 288 mil folhas.

Por outro lado, visto que não existem publicações na literatura sobre modelagem individual de biomassa de Pimenta pseudocaryophyllus, apenas trabalhos semelhantes desenvolvidos para outras espécies nativas de importância comercial, como a araucária, a bracatinga e a canela-amarela (WATZLAWICK et al., 2009; URBANO et al., 2008; BARBEIRO et al., 2009), torna-se relevante novos estudos dessa grandeza.

Igualmente, para facilitar as quantificações de biomassa desta espécie, visando o fornecimento de matérias-primas para a indústria de produtos nãomadeiráveis, como a farmacêutica e a cosmética. Além disso, as folhas de cataia são também utilizadas tradicionalmente para fins medicinais e, mais recentemente, para a produção de aguardente aromatizada, "whisky caiçara", com as folhas da planta, que configura uma atividade econômica e renda adicional assim como, para que se mantenha a tradição das famílias residentes na comunidade.

Recentemente, D'angelis \& Negrelle (2014), também confirmou o potencial econômico e a importância cultural desta espécie para as comunidades tradicionais. Ademais, a indicação dessa potencialidade é importante, ainda, para a conservação e o uso sustentável da espécie.

Sabendo-se do potencial econômico da cataia para a agroindústria de "whisky", bem como a estimativa de biomassa, considerando-se a indicação popular do uso de 10 folhas por garrafa (aproximadamente $4 \mathrm{~g}$ ), tem-se uma estimativa potencial total de 27 mil garrafas nas três UT's consideradas.

Desse modo, considerando o estoque foliar atual estimado para as três UT's, e o número atual de 8 extratores envolvidos no projeto, calcula-se um limite de 87 garrafas por mês, para cada comunitário, representando a extração de 33 quilos de biomassa seca foliar durante o ano.

Entretanto, para que o processo de extração das folhas de $P$. pseudocaryophyllus ocorra de forma sustentável, bem como preserve os processos fisiológicos naturais e regeneração da espécie, recomenda-se um sistema de colheita realizado através da poda de metade dos ramos com folhas, de todos os indivíduos com diâmetro acima de $5 \mathrm{~cm}$ de diâmetro, respeitando-se à alternância das Unidades de Trabalho a cada ano.

\section{CONCLUSÕES}

Os modelos testados apresentaram desempenho razoável para a variável dependente biomassa seca das folhas, sendo que o modelo Stepwise Completo, obteve o melhor resultado para a estimativa de biomassa foliar.

A extração das folhas de $P$. pseudocaryophyllus deve ser manejada de forma sustentável, visando a manutenção da espécie, bem como sua continuidade na atividade econômica e cultural das famílias tradicionais da comunidade de Ilha Comprida.

\section{AGRADECIMENTOS}

Ao Serviço Florestal Brasileiro - Unidade Regional Sul e à Prefeitura da Estância Balneária de Ilha Comprida, pelo apoio logístico e financeiro na condução do estudo.

\section{REFERÊNCIAS}

ANACLETO, A. et al. Extractive traditional rural communities from Atlantic rainforest: an approach on families who live in the territory of the environmental Protection Area of Guaratuba in Paraná Coast - Brazil. Espacios, v.38, n.7, p.4-11, 2017

BARBEIRO, L.S.S. et al. Equações para estimativa da biomassa individual de Nectandra grandiflora Ness (canela-amarela). Floresta, v.39, n.4, p.833-843, 2009.

BECEGATO, J.L.; SUGUIO, K. Impacto ambiental antrópico na APA (Área de Proteção Ambiental) da llha Comprida (SP): Da préhistória à Atualidade. Revista UnG - Geociências, v.6, n.1, p.107117, 2007.

BRASIL. Lei no 9.985, de 18 de julho de 2000. Diário Oficial da União, Brasília, $2000 . \quad$ Disponível em: https://www.planalto.gov.br/ccivil_03/leis/19985.htm 
BRASIL. Lei $n=11.428$, de 22 de dezembro de 2006. Diário Oficial da União, Brasília, 2000. Disponível em: http://www.planalto.gov.br/ccivil_03/_ato2004-2006/2006/lei/ I11428.htm

CORTE, A.P.D. et al. Fator de expansão de biomassa e razão de raízes - parte aérea para Pinus spp. plantadas no Sul do Brasil. Floresta, v.42, n.4, p.755-768, 2012.

D'ANGELIS, A.S.R.; NEGRELLE, R.R.B. Pimenta pseudocaryophyllus (Gomes) Landrum: aspectos botânicos, ecológicos, etnobotânicos e farmacológicos. Revista Brasileira de Plantas Medicinais, v.16, n.3, p.607-617, 2014.

IIZUKA, E.S. et al. Gestão social e cidadania deliberativa: A experiência de Ilha Comprida - São Paulo. Cadernos EBAPE.BR, v.9, n.3, p.748-779, 2011.

PAULA, J.A. et al. Pharmacognostic study about Pimenta pseudocaryophyllus (Gomes) LR Landrum leaves-Myrtaceae. Revista Brasileira de Farmacognosia, v.18, n.2, p.265-278, 2008.

SANTOS, A.J. et al. Produtos não madeireiros: conceituação, classificação, valoração e mercados. Floresta, v.33, n.2, p. 215224, 2003.

SANTOS, V.C. O princípio da subsidiariedade e sua relação com a APA do município de Ilha Comprida, SP. GEOgraphia, v.18, n.37, p.138-153, 2016.

SILVA, J.F. Dados climatológicos de Cananéia e Ubatuba (Estado de São Paulo). Boletim climatológico do Instituto Oceanográfico, v.6, n.1, p.1-21, 1989.

SILVESTRE; R.; BONAZZA, M.; STANG, M.; LIMA, G. C. P.; KOEPSEL, D. A.; MARCO, F. T.; CIARNOSCHI, L. D.; SCARIOT, R.; MORÊS, D. F. Equações volumétricas em povoamentos de Pinus taeda L. no município de Lages-SC. Nativa, v. 02, n. 01, p. 01-05, 2014.

VIANA, H. et al. Predição da Biomassa Aérea da Pinus pinaster Aiton por um Sistema de Equações Aditivas Integrado no Simulador Open Source Modis Pinaster. Silva Lusitana, v.21, (Especial), p.99-109, 2013.

URBANO, E. et al. Modelagem da biomassa aérea em bracatingais nativos da Região Metropolitana de Curitiba. Floresta, v.38, n.2, p.361-372, 2008.

WATZLAWICK, L.F. et al. Estimativa de biomassa e carbono em floresta com Araucária utilizando imagens do satélite Ikonos II. Ciência Florestal, v.19, n.2, p.169-181, 2009.

YOKOMIZO, N.K.S.; NAKAOKA-SAKITA, M. Atividade antimicrobiana e rendimento do óleo essencial de Pimenta pseudocaryophyllus var. pseudocaryophyllus (Gomes) Landrum, Myrtaceae. Revista Brasileira de Plantas Medicinais, v.16, n.3 p.513-520, 2014. 